
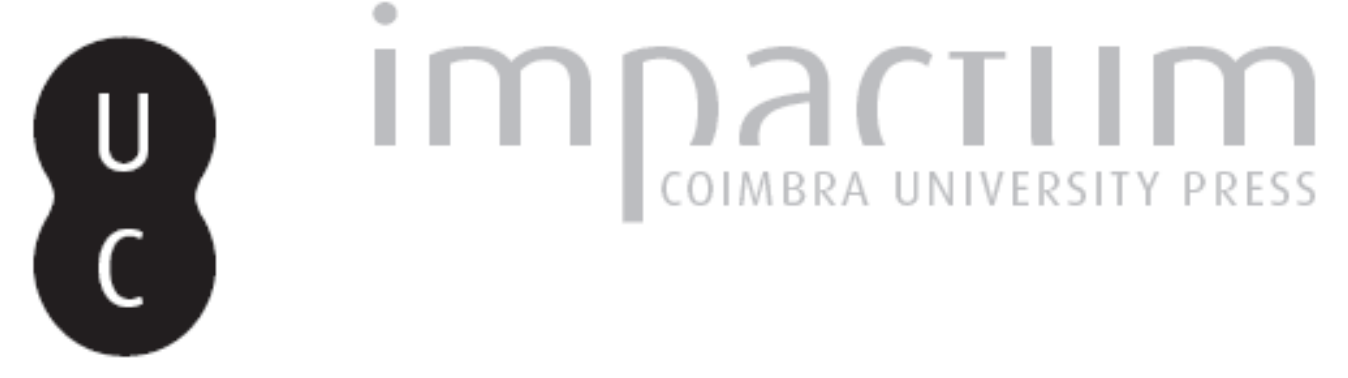

\title{
Influência das características antropométricas maternas no resultado da gravidez, em mulheres de origem cabo-verdiana e portuguesa
}
Autor(es):
Marques, Vítor Rosado
Publicado por: CIAS - Centro de Investigação em Antropologia e Saúde
URL persistente:
URI:http://hdl.handle.net/10316.2/41241
DOI:
DOI:http://dx.doi.org/10.14195/2182-7982_17_5
Accessed : $\quad$ 26-Apr-2023 11:31:17

A navegação consulta e descarregamento dos títulos inseridos nas Bibliotecas Digitais UC Digitalis, UC Pombalina e UC Impactum, pressupõem a aceitação plena e sem reservas dos Termos e Condições de Uso destas Bibliotecas Digitais, disponíveis em https://digitalis.uc.pt/pt-pt/termos.

Conforme exposto nos referidos Termos e Condições de Uso, o descarregamento de títulos de acesso restrito requer uma licença válida de autorização devendo o utilizador aceder ao(s) documento(s) a partir de um endereço de IP da instituição detentora da supramencionada licença.

Ao utilizador é apenas permitido o descarregamento para uso pessoal, pelo que o emprego do(s) título(s) descarregado(s) para outro fim, designadamente comercial, carece de autorização do respetivo autor ou editor da obra.

Na medida em que todas as obras da UC Digitalis se encontram protegidas pelo Código do Direito de Autor e Direitos Conexos e demais legislação aplicável, toda a cópia, parcial ou total, deste documento, nos casos em que é legalmente admitida, deverá conter ou fazer-se acompanhar por este aviso.

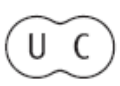




\section{Antropologia Portuguesa}

$16-17 \cdot 1999-2000$

Departamento de Antropologia | Universidade de Coimbra 


\title{
Influência das características antropométricas maternas no resultado da gravidez, em mulheres de origem cabo-verdiana e portuguesa
}

\author{
Vitor Rosado Marques \\ Centro de Antropobiologiz \\ Instituto de Investigação Cientifíca Tropical \\ 1000-219 Lisboa, Portugal \\ vrmarques@mail.telepac.pr
}

\section{Resumo}

O objectivo deste trabalho é avaliar a relação entre as características antropométricas maternas, antes e durante a gravidez, e as dos recém-nascidos, de mulheres de origem cabo-verdiana $(\mathrm{N}=140)$ e portuguesa $(\mathrm{N}=170)$, residentes na Buraca, Amadora. No início da gestação não foram encontradas diferenças estatisticamente significativas na antropometria materna, entre os dois grupos de grávidas, excepto para a estatura. A idade gestacional e as variáveis antropométricas dos recém-nascidos medidas ao nascimento não apresentaram diferenças estatisticamente significativas entre as duas amostras. O tempo de gestação é a variável materna que maior influência tem no resultado da gravidez. Os recém-nascidos cabo-verdianos apresentam uma maior correlação com a antropometria materna do que os portugueses. Nas grávidas portuguesas, o estado nutricional no segundo trimestre é de particular importância para o desenvolvimento fetal. Nas cabo-verdianas, observámos uma correlaçāo entre o peso ao nascimento e a antropometria materna ao longo de toda a gravidez. Igualmente neste grupo, e contrariamente ao que observámos nas grávidas portuguesas, o perimetro cefálico também apresenta uma forte influência da antropometria materna durante a gestaçāo.

Palavras-chave

Gravidez, recém-nascidos, antropometria, Cabo Verde, Portugal, imigração.

\section{Abstract}

The purpose of this study is to understand the relationship between the maternal anthropometrical characteristics, before and during the pregnancy, and the out- 
come of pregnancy, in a sample of women from Cape Vercle $(\mathrm{N}=140)$ and Portugal $(\mathrm{N}=170)$. In the beginning of the pregnancy we did not find statistically differences between the maternal anthropometry in both groups, except for the stature. The gestational age and the size at birth did not show statistically differences between both groups. The duration of gestation has the greater impact on the outcome of pregnancy. The newborns from Cape Verde mothers are more influenced by the maternal anthropometry than the Portuguese. In the Portuguese pregnant women nutritional status is very important for intrauterine growth, particularly in the 2nd trimester. In the pregnant women from Cape Verde we observed a positive correlation between the maternal anthropometry during the whole gestational period with the birth weight. In the same way, and contrary to the observed in Portuguese, head circumference is also influenced by the maternal anthropometry during the whole gestational period.

\section{Keywords}

Pregnancy, newborns, anthropometry, Cape Verde, Portugal, immigration.

\section{Introdução}

A partir do momento da concepção, iniciam-se na mulher profundas e múltiplas alterações fisiológicas em todos os sistemas orgânicos, alterações que se continuam a manifestar ao longo de toda a gravidez e, algumas delas, persistem mesmo após o parto.

O grande aumento da capacidade uterina é acompanhado por um crescimento do tecido muscular e dos vasos sanguíneos de modo a proporcionar um aumento do fluxo sanguíneo necessário à nutrição do feto.

Verifica-se, igualmente, um aumento do volume total de água corporal e uma grande acumulação de gordura corporal indispensável para suprir as necessidades metabólicas da gestação e, posteriormente, utilizável durante o período de aleitamento.

Todas estas alterações, características deste período particular da vida reprodutiva da mulher, manifestam-se, geralmente, por um grande aumento de peso. Assim, a influência da quantidade de gordura materna no resultado da gravidez tem, também, sido alvo de interpretações, por vezes contraditórias, quer em relação à sua influência directa, quer em relaçāo à sua variação ao longo da gravidez, nomeadamente acerca dos momentos em que essa variação ocorre. 
Enquanto que para muitos autores o ganho de peso materno é um importante indicador do peso ao nascimento (Alberti-Fidanza et al., 1998; Hediger et al., 1990; Jiménes e Bacallao, 1995; Langhoff-Ross et al., 1987; School et al., 1989; Stevens-Simon e McAnarney, 1988), outros há que não encontraram nesse aumento ponderal um factor de grande influência no peso do recém-nascido (Dawes e Grudzinskas, 1991; Ricalde et al., 1998). Já em relação ao peso antes da gravidez há um unanimismo quase generalizado quanto à sua influência no peso ao nascimento entre os diferentes investigadores.

Este estudo tem por objectivo identificar, em duas amostras de mulheres pertencentes a um nível socioeconómico baixo, porém de origem diferente (portuguesa e cabo-verdiana, residentes em Portugal), quais as características antropométricas maternas, no início e ao longo da gravidez, que melhor se relacionam com a antropometria dos respectivos recém-nascidos.

\section{Metodologia}

A recolha dos dados realizou-se no Centro de Saúde da Reboleira Extensão da Buraca (Amadora), entre 1993 e 1996. Foram estudadas 310 grávidas e igual número de recém-nascidos, 140 de origem cabo-verdiana e 170 de origem portuguesa. As variáveis antropométricas maternas estudadas foram a estatura (Est), o peso, o ganho total de peso durante a gravidez (GTP), o índice de massa corporal (IMC), a circunferência do braço (CB), a prega cutânea tricipital (PCT) e a prega cutânea subescapular (PCS). Posteriormente, foram calculados os índices: área adiposa do braço (AAB), área muscular do braço (AMB), área total do braço (ATB) e o índice de adiposidade do braço (IAB). Todas as mediçōes foram efectuadas pelo autor, de acordo com o Programa Biológico Internacional (Weiner e Lourie, 1981).

No caso dos recém-nascidos utilizaram-se as medidas efectuadas na maternidade, pelo pessoal médico ou de enfermagem (PNas - peso ao nascimento, CNas - comprimento ao nascimento e PCefNas - perímetro cefálico ao nascimento), aquando do parto e registadas no Boletim Individual de Saúde da criança. 
A idade gestacional (IG) foi calculada como sendo o intervalo entre o primeiro dia do último período menstrual e o parto, medido em semanas completas. Sempre que este valor levantava dúvidas, era confirmado pela estimativa feita através de ecografia realizada cerca das 18-20 semanas.

Sendo o peso a variável que maior alteração sofre durante a gravidez, utilizou-se apenas o valor da primeira medição efectuada até às 13 semanas de gestação (limite do primeiro trimestre) que, segundo o Institute of Medicine (1980), referido por WHO (1995), pode ser utilizado em alternativa ao valor pré gestacional, quando este não está disponivel. Também para as outras variáveis referentes ao início da gravidez utilizou-se o valor da primeira medição efectuada no primeiro trimestre.

\section{Análise estatística}

As variáveis descontínuas foram estudadas através de uma Análise $c^{2}$ (Pearson). Calcularam-se as suas frequencias quer em valor absoluto, quer em percentual. A comparação entre as médias das variáveis das amostras foi feita através de um Teste $t$ de Student para amostras independentes. $\mathrm{Na}$ análise das relações entre as variáveis maternas e as dos recém-nascidos, utilizou-se a correlação de Pearson no caso de existir uma distribuição normal e a da Spearman quando esta condição não se verificou.

\section{Resultados e Discussão}

A grande maioria das mulheres estudadas é casada (ou vive em união de facto), tem menos de 9 anos de escolaridade, faz parte da população activa e vive em bairros de construção clandestina, posteriormente legalizados, ou em bairros de realojamento com evidentes sinais de degradação (tabela 1). Pertencem a um estrato socioeconómico baixo com fracos rendimentos familiares. Cerca de $60 \%$ das famílias têm um rendimento familiar mensal inferior a $750 €$ e perto de $30 \%$ mesmo abaixo dos $500 €$.

A idade média materna é semelhante nos dois grupos estudados e ronda os 26 anos, no entanto, nas mulheres cabo-verdianas existe uma maior dispersão dos valores, com uma variância e um desvio padrão maiores.

No início da gravidez não foram encontradas diferenças estatisticamente significativas, entre os dois grupos de grávidas, em relação às va- 
riáveis antropométricas estudadas, com excepçāo para a estatura, que apresenta um valor médio mais elevado nas cabo-verdianas (tabela 2).

Tabela 1. Caracteristicas socio-demográficas das mulheres estudadas, de acordo com a sua origem.

\begin{tabular}{|c|c|c|c|c|c|c|}
\hline \multirow{2}{*}{ Variáveis } & \multicolumn{2}{|c|}{ Portuguesas } & \multicolumn{2}{|c|}{ Cabo-verdianas } & \multirow{2}{*}{$x^{2}$} & \multirow{2}{*}{$\mathrm{p}$} \\
\hline & $N$ & $\%$ & $\mathrm{~N}$ & $\%$ & & \\
\hline Estado civil & & & & & 10,199 & W: \\
\hline Casada/Uniāo de facto & 151 & 88,80 & 105 & 75,00 & & \\
\hline Solteira & 19 & 11,20 & 35 & 25,00 & & \\
\hline Residência & & & & & 132,813 & **k \\
\hline Bairro Cova da Moura & 24 & 14,10 & 111 & 79,30 & & \\
\hline Bairro do Zambujal & 67 & 39,40 & 15 & 10,70 & & \\
\hline Outro & 79 & 46,50 & 14 & 10,00 & & \\
\hline Nivel de escolaridade & & & & & 36,345 & ****: \\
\hline$<4$ anos & 0 & 0,00 & 16 & 11,90 & & \\
\hline $4-6$ anos & 84 & 50,00 & 83 & 61,50 & & \\
\hline 9 anos & 47 & 28,00 & 28 & 20,70 & & \\
\hline$\geq 12$ anos & 37 & 22,00 & 8 & 5,90 & & \\
\hline Estatuto profissional & & & & & 3,938 & $: *$ \\
\hline Trabalhadora & 120 & 71,40 & 85 & 60,70 & & \\
\hline Nāo trabalhadora & 48 & 28,60 & 55 & 39,30 & & \\
\hline
\end{tabular}

O peso no início da gravidez era também mais elevado nas grávidas cabo-verdianas, embora as diferenças não fossem estatisticamente significativas, porém, o ganho total de peso foi estatisticamente mais elevado nas grávidas portuguesas.

A maioria das grávidas de ambos os grupos apresentava um estado nutricional normal, estimado a partir do Índice de Massa Corporal - IMC (Institute of Medicine, 1990, in Fields et al., 1997; Keppel e Taffel, 1993; Kolasa e Weismiller, 1997), não se tendo encontrado diferenças estatisticamente significativas entre os grupos (tabela 2).

Os Z-scores da maioria das variáveis antropométricas medidas no início da gravidez apresentam, em relação aos valores médios de referência do National Center for Health Statistics - NCHS, afastamentos que não excedem um desvio padrão (Marques, 1999). 


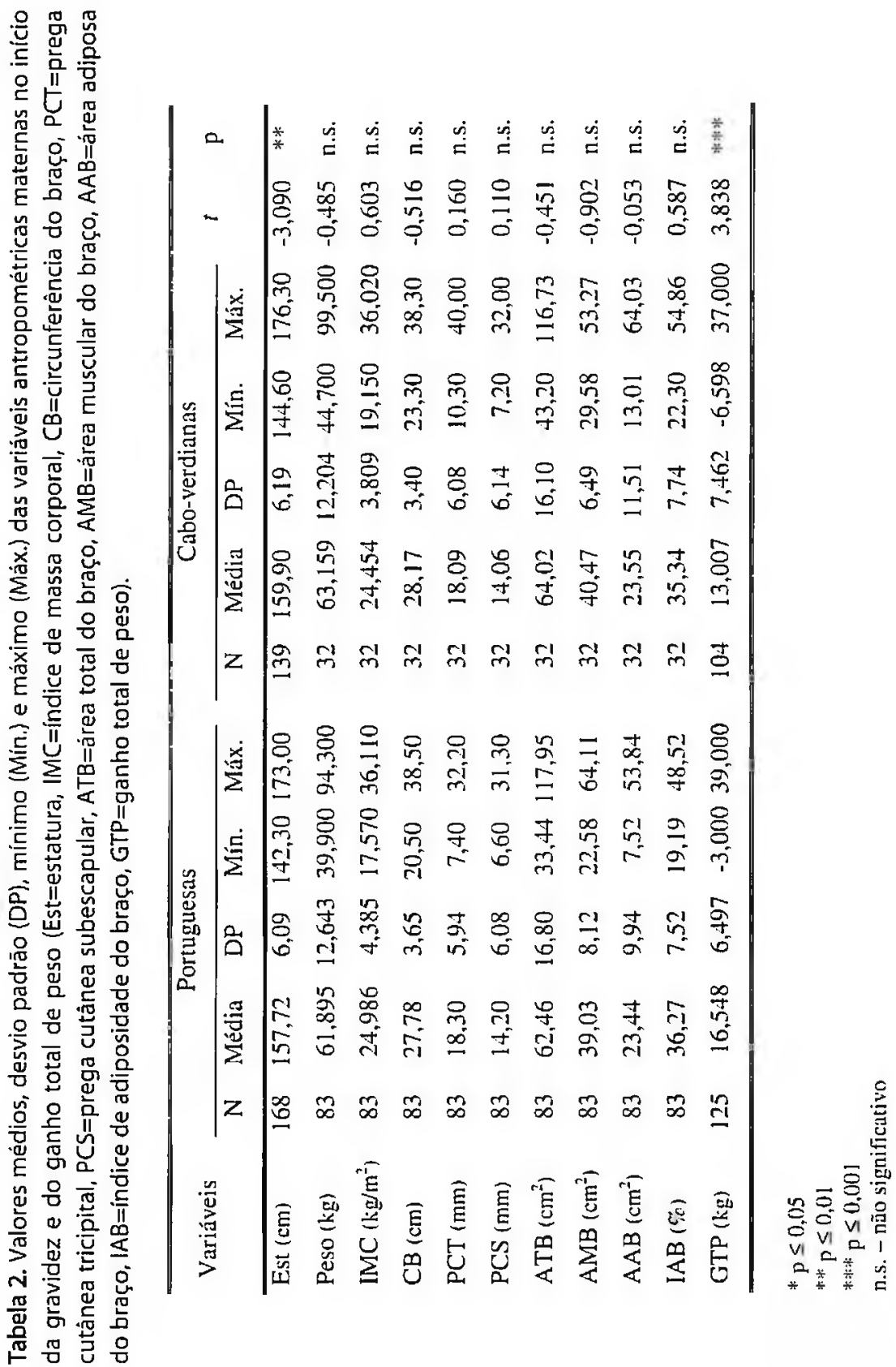


Apesar de não termos encontrado diferenças estatisticamente significativas no que respeita à idade média materna nas mulheres estudadas, observou-se uma frequência de multiparas significativamente mais elevada nas cabo-verdianas do que nas portuguesas, o que se deve a uma menor idade ao casamento e ao nascimento do primeiro filho (Marques, 1999). Verificou-se que, em ambos os grupos, a maioria das variáveis antropométricas maternas medidas no início da gestaçāo tinha valores significativamente mais elevados nas grávidas multíparas (tabela 3).

Os valores médios da idade gestacional e das variáveis antropométricas ao nascimento dos recém-nascidos de ambos os grupos não apresentaram diferenças estatisticamente significativas (tabela 4), quer quando comparamos os sexos reunidos, quer quando a comparação é feita dentro do mesmo sexo, o que vem confirmar resultados obtidos em anterior estudo (Marques, 1990).

O facto de não encontrarmos diferenças estatisticamente significativas para o peso dos recém-nascidos nas amostras consideradas, vem confirmar o que anteriormente referimos, por um lado, a existência de um estado nutricional semelhante nas grávidas portuguesas e nas cabo-verdianas, uma vez que o peso ao nascimento é o principal indicador do estado nutricional materno. Por outro lado, sendo o peso ao nascimento muito sensível ao ambiente materno, vem também confirmar a similaridade entre os grupos de grávidas estudados. No entanto, os recém-nascidos do sexo masculino são maiores do que os do sexo feminino, sendo as diferenças estatisticamente significativas para o comprimento ( $\mathrm{CNas}$ ) e para o perímetro cefálico ao nascimento (PCefNas) nos portugueses (tabela 5) e nos caboverdianos apenas o perímetro cefálico é significativamente maior (tabela 6). Excluindo da análise os recém-nascidos de prétermo (IG $<37$ semanas), que são em reduzido número (cerca de $4 \%$ ), apenas a diferença no comprimento ao nascimento já existente entre os dois sexos nos recémnascidos cabo-verdianos, se torna estatisticamente significativa.

Estes resultados são discordantes dos encontrados por Cameron et al. (1998) e Johnson et al. (1994) num estudo realizado em mulheres afroamericanas, onde se verificaram diferenças entre os sexos apenas em relação ao peso ao nascimento. No entanto, Frisancho et al. (1977), numa população urbana do Peru, observaram valores significativamente maiores para todas as variáveis dos recém-nascidos do sexo masculino, com excepção da idade gestacional. 


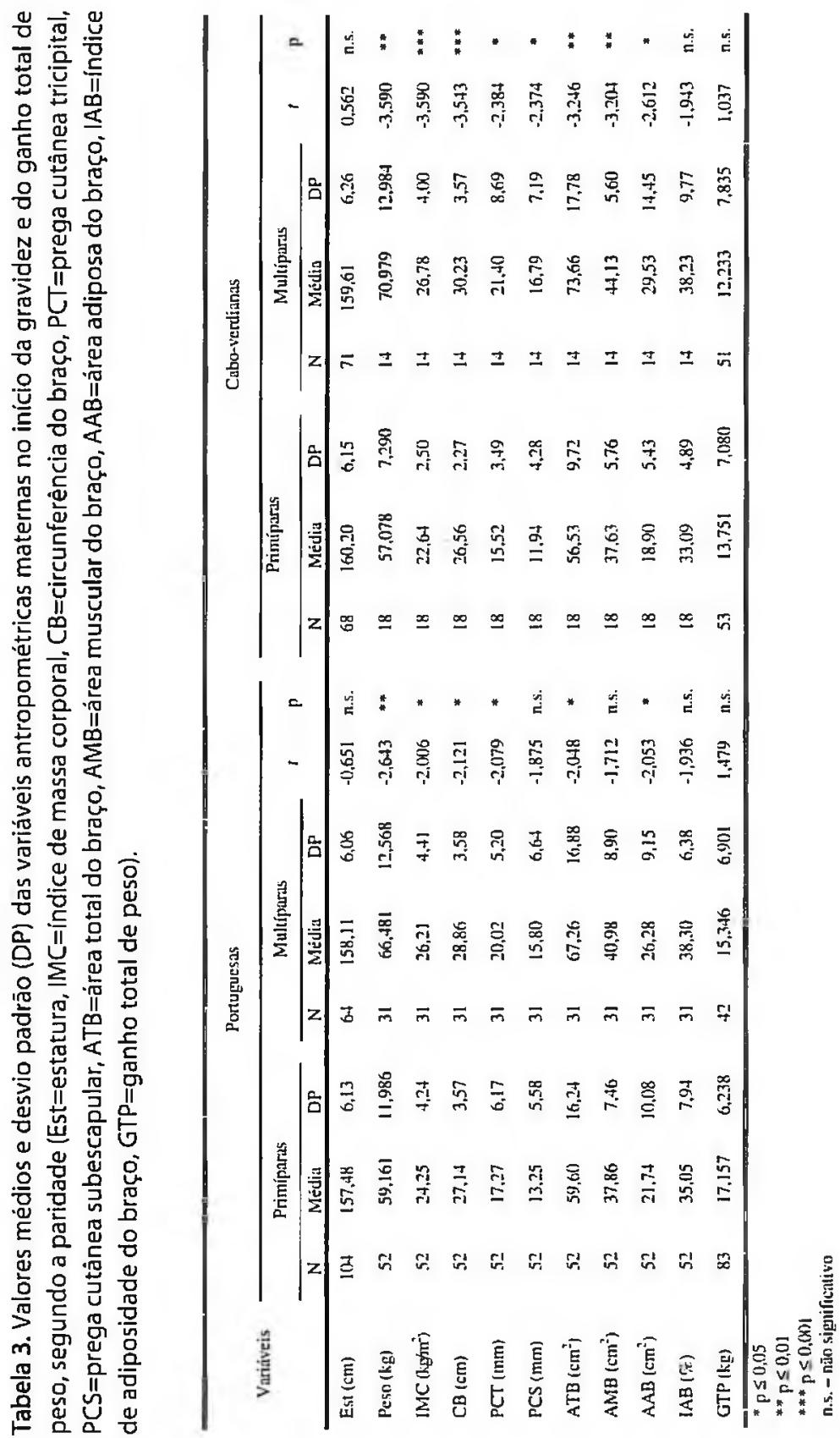



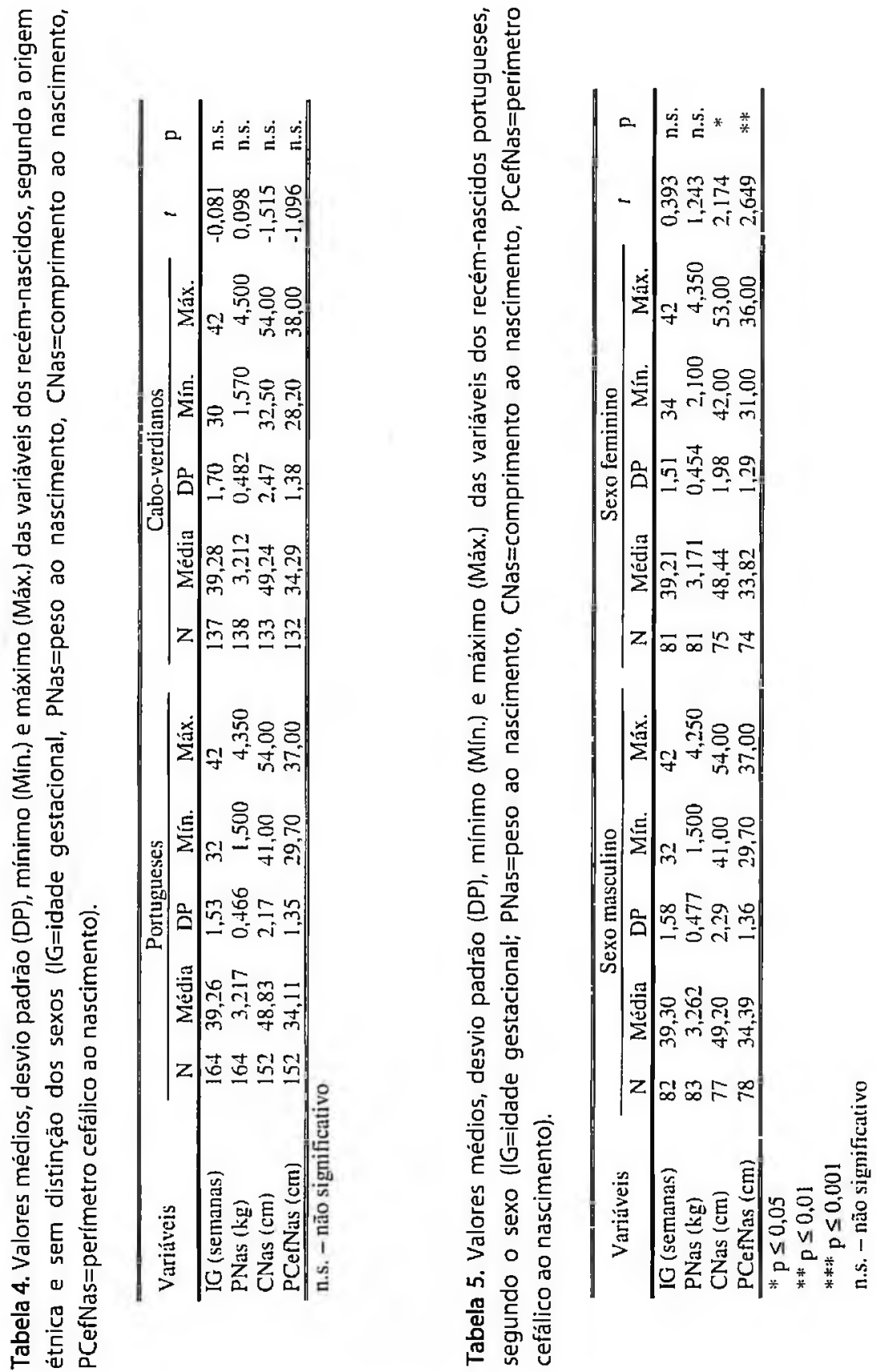
Quando foi considerada a paridade, estes resultados não sofreram alteração relevante, ou seja, não foram encontradas diferenças estatisticamente significativas entre as variáveis antropométricas dos recém-nascidos portugueses e cabo-verdianos filhos de mães primíparas, o mesmo sucedendo com os filhos de mães multíparas. No entanto, os recém-nascidos cabo-verdianos filhos de mães multíparas apresentavam um peso ao nascimento (PNas) significativamente superior ao dos filhos de mães primíparas (tabela 7).

Tal como foi observado em anteriores estudos (Dawes e Grudzinkas, 1991; Ricalde et al., 1998), o tempo de gestação (TG) é a variável que maior influência tem no resultado da gravidez, apresentando, nos grupos estudados, uma correlação positiva e significativa com todas as medidas antropométricas do recém-nascido (tabelas 8 e 9).

Observam-se, ainda, correlaçōes positivas e significativas de diversas variáveis antropométricas maternas, medidas no início da gravidez, com a antropometria dos recém-nascidos, sendo esta relação mais evidente nos recém-nascidos de origem cabo-verdiana (tabela 8). Assim, é possível observar neste grupo uma relação significativa do peso materno com todas as variáveis do recém-nascido e da estatura (Est) com o peso (PNas) e o comprimento ao nascimento (CNas). Também a prega cutânea tricipital (PCT), que é referida por Neggers et al. (1992) como sendo um óptimo indicador do peso ao nascimento, a área adiposa do braço (AAB) e o índice de adiposidade do braço (IAB) mostraram correlaçōes positivas e significativas com o peso e o perímetro cefálico ao nascimento.

Os recém-nascidos de origem portuguesa parecem mostrar uma maior independência em relação à antropometria materna (tabela 9), verificando-se apenas uma correlação estatisticamente significativa entre a estatura materna e o peso ao nascimento. Em relação ao comprimento e ao perímetro cefálico ao nascimento não foi encontrada qualquer correlação significativa com nenhuma das variáveis antropométricas maternas.

O facto de não encontrarmos uma relação significativa entre o peso materno no início da gravidez e o peso ao nascimento neste grupo está em contradição com os resultados da maioria dos autores (Backstrand, 1995; Dawes e Grudzinskas, 1991; Karim e Mascie-Taylor, 1997; Kirchengast e Hartmann, 1998; Neumann et al., 1995; Neyzi et al., 1987; Ricalde et al., 1998; Smith, 1997). Contudo, o peso medido no segundo e no terceiro trimestres já se apresenta associado com o peso e o comprimento ao nascimento. 

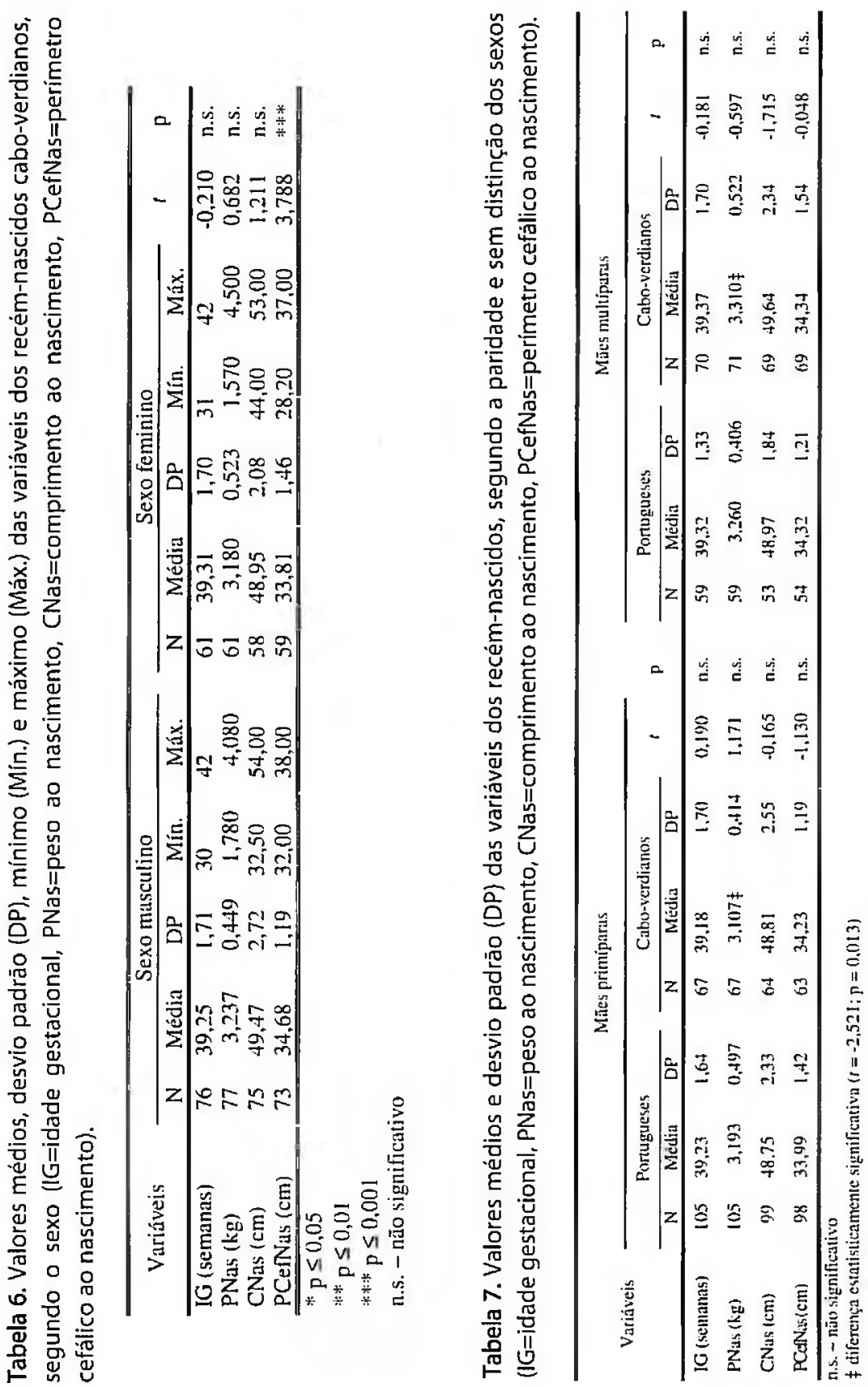
畩

高鮞

ำ 음

范

$\geqslant 5$

藏

는

愛

$\frac{n}{4}$

要

要

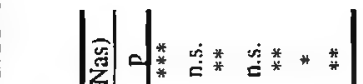

熍

穷

응

焉

है

믕

亭

음 든

:

空

in

(1)

ทั $\frac{\mathrm{g}}{\mathrm{n}}$

을음

要

要

d

O $E$

वे

$\circ$ 든

을 氜

焉

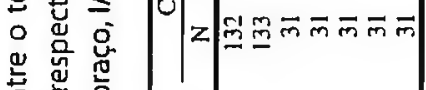

密

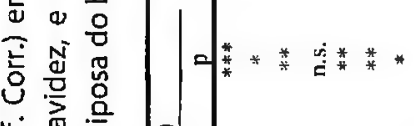

낭 음

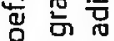

记

요

造里

जิ

S

ง

II 음

䓵出

苟 气 啚

문 믄

㟧苋

○苗

를

\&

○ ․ㅣㄴ

थ

突 总

-

능 苍

บ

प्ष

空 은

贾

急家员

空 员

은

过罂高

过 음

$\stackrel{\mathscr{C}}{\mathrm{E}}$

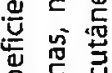

讨 茜

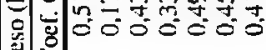

을 음

岌. 而

运 은

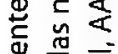

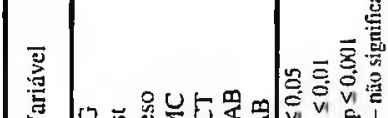

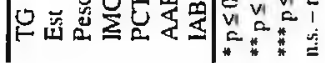

$\infty$ 远

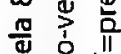

高员苋

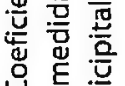

ơ

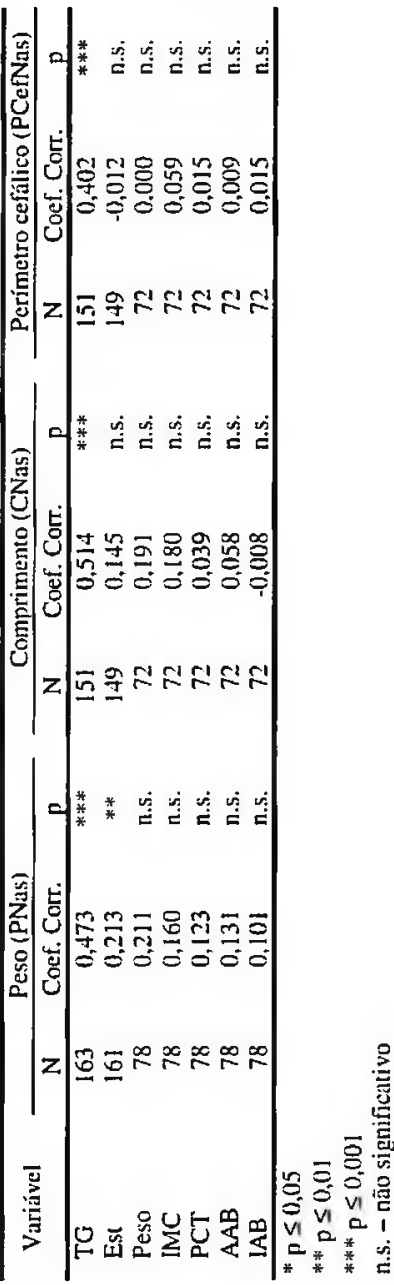


O ganho total de peso (GTP) durante a gravidez, que tinha valores significativamente mais elevados nas mulheres portuguesas, apresenta-se correlacionado com o peso ao nascimento, o que mostra a importância deste factor no crescimento intra-uterino observada por diversos autores (Jiménez e Bacallao, 1995; Smith, 1997), referindo, ainda, Stevens-Simon e McAnarney (1992) a vantagem de poder ser modificado durante a gravidez. Kirchengast e Hartmann (1998) realçam, também, a importância do ganho de peso para o resultado da gravidez, mas concluem que elevados ganhos de peso em mulheres de baixo nivel ponderal antes da gravidez não são suficientes para compensar o efeito negativo do baixo peso antes da gravidez. Pelo contrário, Ricalde et al. (1998) não encontraram qualquer associação entre o ganho de peso e o peso ou o comprimento ao nascimento, justificando este facto pela provável independência do ganho de peso em relação ao estado nutricional antes da gravidez.

Com excepção do peso materno, as alteraçōes que as variáveis maternas sofrem entre os momentos estudados não apresentam qualquer correlação com as medidas ao nascimento (Marques, 1999). No entanto, as medições efectuadas nesses momentos da gravidez, particularmente no segundo e terceiro trimestres, apresentam-se positiva e significativamente correlacionadas com a antropometria do recém-nascido. Assim, verifica-se nas grávidas portuguesas correlações estatisticamente significativas entre as medições do segundo trimestre e o peso e o comprimento do recém-nascido (tabela 10 ), enquanto que nos recém-nascidos filhos de mães cabo-verdianas o peso e o perímetro cefálico ao nascimento apresentam-se correlacionados, com as medidas antropométricas maternas tanto ao segundo, como ao terceiro trimestres (tabela 11).

Destes resultados é de realçar a independência manifestada pelo perímetro cefálico, no caso dos recém-nascidos portugueses e do comprimento nos cabo-verdianos em relação às características maternas durante a gravidez, sendo, então, a sua variabilidade explicada, provavelmente, através de outros factores de ordem genética e/ou ambiental.

As diferenças observadas nos dois grupos de grávidas, em relação às variáveis indicadoras da adiposidade subcutânea e tendo em consideração que durante a gestação se observa um decréscimo dos valores médios da maioria destas variáveis nas mulheres cabo-verdianas (Marques, 1999), leva-nos a sugerir que estas grávidas utilizam a gordura subcutânea armazenada na regiāo tricipital sobretudo nas necessidades exigidas pelo 


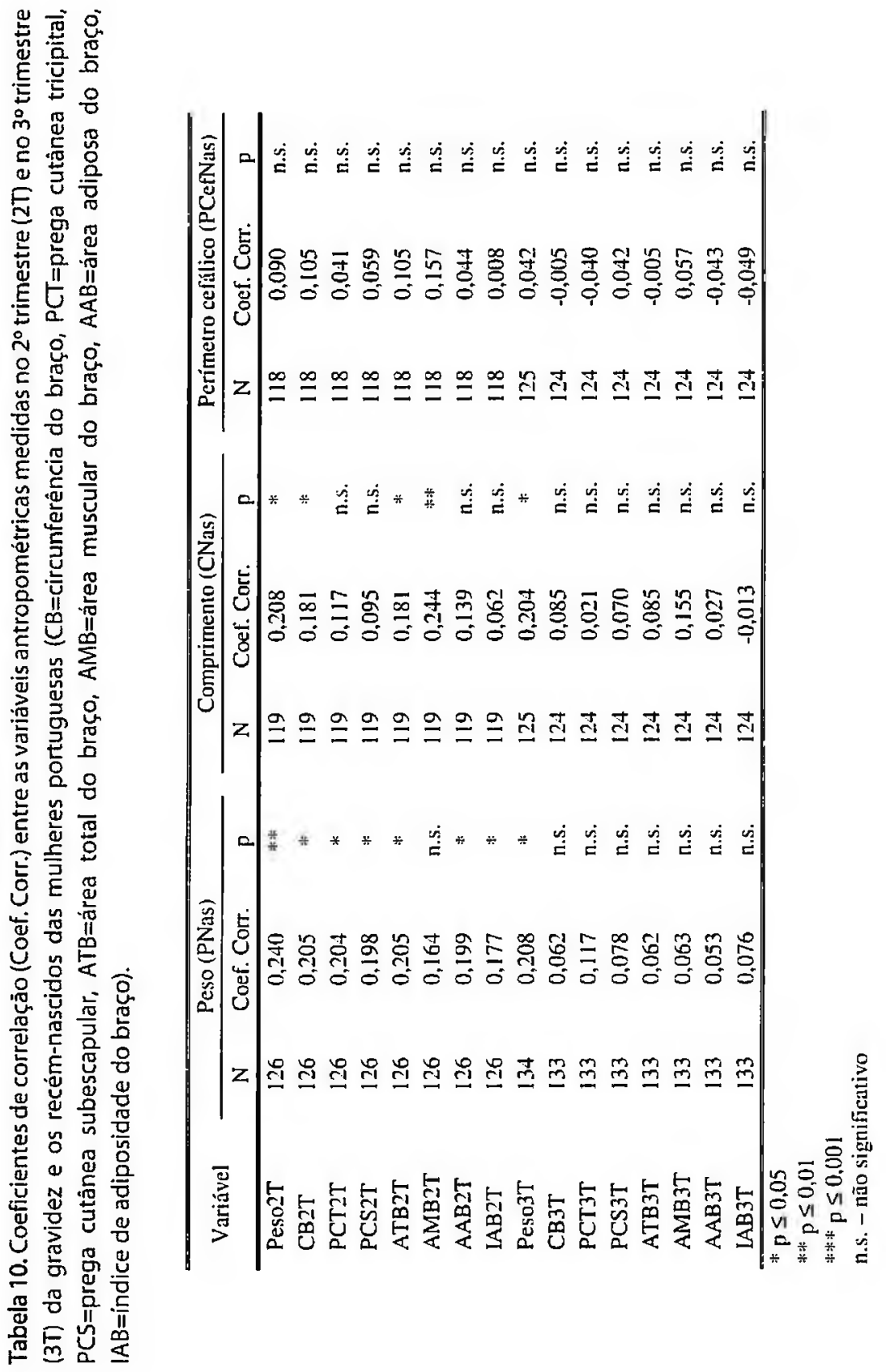




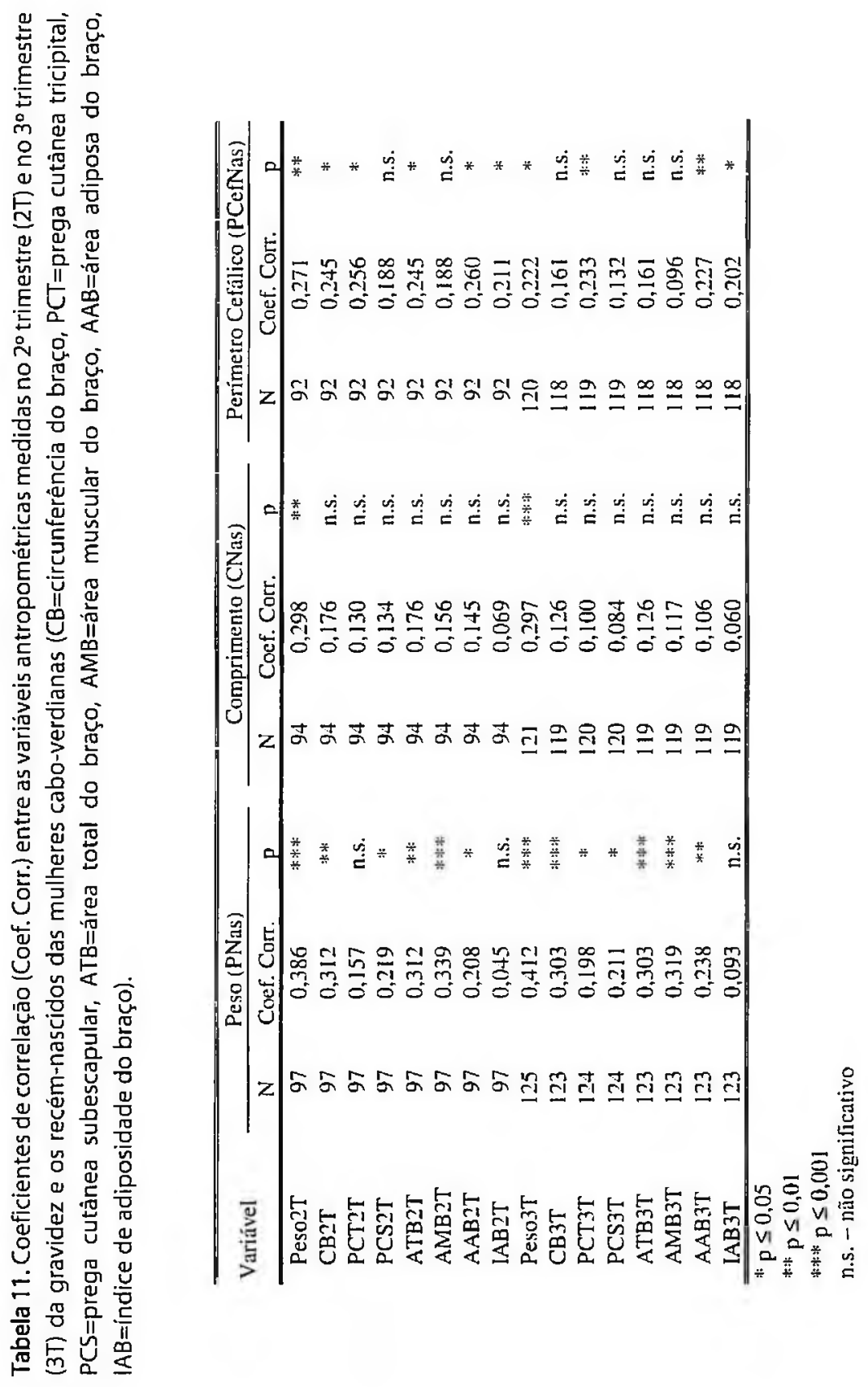


crescimento fetal, enquanto nas grávidas portuguesas essa reserva é utilizada mais tarde, provavelmente, no processo da lactação.

$O$ facto de apenas nos filhos das mulheres cabo-verdianas existir uma relação significativa das variáveis maternas medidas no terceiro trimestre, poderá estar relacionado com o modo como estas variáveis se comportam ao longo da gravidez, uma vez que neste grupo de gestantes as alterações morfológicas maternas se prolongam até mais tarde, enquanto que nas portuguesas a maioria das alteraçōes ocorre, preferencialmente, no segundo trimestre.

\section{Conclusão}

As características antropométricas maternas estudadas no início da gravidez, à excepção da estatura que é mais elevada nas mulheres cabo-verdianas, não apresentaram diferenças estatisticamente significativas entre os dois grupos de gestantes, o que poderá dever-se ao facto de estas mulheres pertencerem a um nível socioeconómico baixo, que não permite que estas diferenças se manifestem.

Ainda que não tenham sido encontradas diferenças significativas entre o peso inicial das grávidas cabo-verdianas e o das portuguesas, observa-se um maior ganho de peso durante a gravidez por parte destas últimas, que apresentam um ganho de peso médio um pouco superior em relação aos valores recomendados pelo Institute of Medicine.

Apesar das mulheres da nossa amostra não viverem, de um modo geral, em condições que pudéssemos considerar boas, elas apresentavam um nível nutricional dentro dos padrões normais e, provavelmente por isso, as suas gravidezes tiveram resultados favoráveis.

O resultado da gravidez é, nas mulheres da nossa amostra, independente da origem étnica, uma vez que não foram encontradas diferenças estatisticamente significativas entre os recém-nascidos de origem portuguesa e de origem cabo-verdiana, para nenhuma das variáveis estudadas.

Uma forte miscigenação com uma acentuada influência portuguesa, aliada a uma integração sem grandes sobressaltos na sociedade de acolhimento, e em que o processo de aculturaçāo aproxima as mulheres cabo-verdianas das portuguesas com um nível socioeconómico semelhante, podem justificar, pelo menos em parte, estes resultados. 
Os recém-nascidos portugueses do sexo masculino apresentam um comprimento e um perímetro cefálico significativamente maiores, em comparação com os do sexo feminino. Nos recém-nascidos cabo-verdianos apenas o perímetro cefálico se apresenta significativamente maior nos do sexo masculino.

O tempo de gestação (TG) é a variável com maior influência na antropometria do recém-nascido, tendo-se observado uma correlação positiva e significativa desta variável com todas as medidas antropométricas do recém-nascido.

Nas grávidas cabo-verdianas observa-se, igualmente, uma correlação do peso com todas as variáveis do recém-nascido, da estatura com o peso e o comprimento ao nascimento e das variáveis indicadoras da composição corporal com o peso e o perímetro cefálico ao nascimento, enquanto nas portuguesas apenas a estatura e o ganho de peso estão correlacionados com o peso ao nascimento.

A interpretação destes resultados poderá indicar que as grávidas cabo-verdianas se adaptaram melhor às condições socioeconómicas desfavoráveis, seja por razōes culturais, seja por razões biológicas, o que permitiu que os factores morfológicos maternos se manifestassem. Todavia, para confirmar esta hipótese será necessário um estudo mais aprofundado sobre os aspectos bioculturais ligados à sexualidade destas mulheres, particularmente no que se refere aos seus comportamentos reprodutivos.

A análise da influência da antropometria materna ao longo da gravidez nas medidas dos recém-nascidos permite-nos concluir que, nas grávidas portuguesas, o estado nutricional no segundo trimestre é de particular importância para o desenvolvimento fetal, estando o peso e o comprimento ao nascimento fortemente correlacionados com as medidas antropométricas maternas neste trimestre, pois que corresponde ao período onde se verifica um maior crescimento linear. No caso das grávidas cabo-verdianas, a situação é diferente, pois observamos uma correlação do peso ao nascimento com a antropometria materna ao longo de toda a gravidez, o que poderá estar associado com o facto de nestas mulheres as alterações morfológicas ocorridas durante a gravidez se prolongarem até mais tarde. Do mesmo modo, e contrariamente ao que observamos nas grávidas portuguesas, o perímetro cefálico ao nascimento também manifesta uma forte influência da antropometria materna durante a gravidez. Pelo contrário, o comprimento apenas está relacionado com o peso mater- 
no durante a gravidez, mostrando assim uma quase total independência em relação ao ambiente materno, podendo-se então concluir que o comprimento dos recém-nascidos cabo-verdianos reflecte, sobretudo, as características genéticas parentais.

Este trabalho pretendeu contribuir para o conhecimento das condições em que ocorre, no nosso país, a gravidez numa população imigrada. No entanto, temos consciência de que um trabalho desta natureza deveria ser complementado com estudos realizados em Cabo Verde, para assim podermos inferir, de um modo mais preciso, acerca das modificações comportamentais ao nível do processo reprodutivo e da gravidez e dos factores que neles interferem. No mesmo sentido, também existe a lacuna de estudos de âmbito nacional, que abordem amostras representativas da população portuguesa e onde se possa analisar a influência dos factores socioeconómicos.

\section{Agradecimentos}

Este trabalho teve o apoio de António Pacheco Agostinho, Lda.; LRC, Laboratórios, Lda.; Laboratoires EXPANSCIENCE PHAR; Nestlé Portugal, S.A. e Rotafar, Representações e Comércio, Lda..

Os meus agradecimentos para todo o pessoal técnico e administrativo do Centro de Saúde da Buraca e um Muito Obrigado para todas as mulheres que participaram neste estudo.

\section{Bibliografia}

Alberti-Fidanza, A.; Parizkova, J.; Fruttini, D. 1998. Changes in anthropometric variables and fat pattern during pregnancy and their relationship with newborn value. Acta Medica Auxologica, 30 (1): 19-30.

Backstrand, J. R. 1995. Maternal anthropometry as a risk predictor of pregnancy outcome: the nutrition CRSP in Mexico. Bulletin of the Wortd Health Organization, 73, Supplement: 96-98.

Cameron, N.; Wet, T. De; Ellison, G. T.; Bogin, B. 1998. Growth in height and weight of South African urban infants from birth to five years: the birth to ten study. American Journal of Human Biology, 10 (4): 495-504. 
Dawes, M. G.; Grudzinskas, J. G. 1991. Repeated measurement of maternal weight during pregnancy. Is this a useful practice? British Journal of Obstetrics and Gynaecology, 98: 189-194.

Fields, S. J.; Livshits, G; Merlob, P.; Sirotta, L. 1997. On the relationship between pregnancy weight gain and chorioamnionitis. American Joumal of Human Biology, 9: 545-553.

Frisancho, A. R.; Klayman, J. E.; Matos, J. 1977. Influence of maternal nutritional status on prenatal growth in a Peruvian urban population. American Journal of Physical Anthropology, 46: 245-274.

Hediger, M. L.; Scholl, T. O.; Ances, I. G.; Belsky, D. H.; Salmon, R. W. 1990. Rate and amount of weight gain during adolescent pregnancy: associations with maternal weight-for-height and birth weight. American Joumal of Clinical Nutrition, 52: 793-799.

Hytten, F. E. 1980. Nutritional aspects of human pregnancy. In: Aebi, H.; Whitehead, R. (ed). Maternal Nutrition during Pregnancy and Lactation. Bern, Verlag Hans Huber; 1: 27-38.

Jiménez, R.; Bacallao, J. 1995. Prognostic performance of several anthropometric indicators for predicting low and insufficient birth weight. American Journal of Human Biology, 7 (3): 303-311.

Johnson, A. A.; Knigth, E. M.; Edwards, C. H.; Oyemade, U. J.; Westney, L. S.; Laryea, H.; Jones, S. 1994. Dietary intakes, anthropometric measurements and pregnancy outcomes. Journal of Nutrition, 124, Supplement (6S): 936S942S.

Karim, E; Mascie-Taylor, C. G. N. 1997. The association between birthweight, sociodemographic variables and maternal anthropometry in a urban sample from Dhaka, Bangladesh. Amnals of Human Biology, 24 (5): 387-401.

Keppel, K. G.; Taffel, S. M. 1993. Pregnancy-related weight gain and retention: implications of the 1990 Institute of Medicine Guidelines. Anerican Joumal of Public Health, 83 (8): 1100-1 103.

Kirchengast, S.; Hartmann, B. 1998. Maternal prepregnancy weight status and pregnancy weight gain as major determinants for newborn weight and size. Amals of Human Biology, 25 (1): 17-28.

Kolasa, K. M.; Weismiller, D. G. 1997. Nutrition during pregnancy. American Family Physician, 56 (1): 205-212.

Langhoff-Ross, J.; Lindmark, G.; Gebre-Medhin, M. 1987. Maternal fat stores and fat accretion during pregnancy in relation to infant birthweight. British Joumal of Obstetrics and Gynaecology, 94: 1170-1177. 
Marques, V. M. R. 1990. Estudo dos efeitos do tabagismo e outros factores externos no recém-nascido. In: Relatório de Actividades desenvolvidas durante o periodo de estágio (1987-1990). Lisboa, Centro de. Antropobiologia Instituto de Investigação Científica Tropical: 45-104.

Marques, V. M. R. 1999. A gravidez na mulher cabo-verdiana e portuguesa. Dissertação apresentada no âmbito das Provas de Acesso à Categoria de Investigador Auxiliar. Lisboa, Centro de Antropobiologia - Instituto de Investigaçāo Científica Tropical.

Neggers, Y; Goldenberg, R. L.; Cliver, S. P.; Hoffman, H. J.; Cutter, G. R. 1992. Usefulness of various matemal skinfold measurements for predicting newborn birth weight. Joumal of the American Dietetic Association, 92 (11): 1393-1394. Neumann, C.; Ferguson, L.; Bwibo, N. O. 1995. Maternal anthropometry as a risk predictor of pregnancy outcome: the nutrition CRSP in Kenya. Bulletin of the World Health Organization, 73, Supplement: 91-95.

Neyzi, O.; Gunoz, H.; Çelenk, A.; Dindar, A.; Bundak, R.; Saglan, H. 1987. Relationships between some maternal factors and pregnancy outcome. Human Biology, 59 (3): 387-398.

Ricalde, A. E.; Velásquez-Meléndez, G.; Tanaka, A. C. d'A.; Siqueira, A. A. F. 1998. Mid-upper arm circumference in pregnant women and its relation to birth weight. Revista de Saúde Puiblica, 32 (2): 112-117.

School, T. O.; Hediger, M. L.; Salmon, R. W.; Belsky, D. H.; Ances, I. G. 1989. Influence of pregnant body mass and weight gain for gestation on spontaneous preterm delivery and duration of gestation during adolescent pregnancy. American Joumal of Human Biology, 1 (6): 657-664.

Smith, C. 1997. The effect of maternal nutritional variables on birthweight outcomes of infants born to Sherpa women at low and high altitudes in Nepal. American Journal of Human Biology, 9: 751-763.

Stevens-Simon, C.; McAnarney, E. R. 1988. Adolescent maternal weight gain and low birth weight: a multifactorial model. American Joumal of Clinical Nutrition, 47: 948-953.

Stevens-Simon, C.; McAnarney, E. R. 1992. Determinants of weight gain in pregnant adoslescents. Joumal of the American Dietetic Association, 92 (11): 1348-1351.

Weiner, J. S.; Lourie, J. A. 1981. Human Biology. A Guide to Field Methods. Oxford, Blackwell Scientific Publications.

World Health Organization 1995. Physical Status: The Use and Interpretation of Anthropometry. Geneva, WHO Technical Report Series, 854, 453 pp. 